Journal of Applied Fluid Mechanics, Vol. 9, Special Issue 2, pp. 207-212, 2016.

Selected papers from the XIIth Franco - Quebec Inter-University

Symposium on Thermal Systems -2015

Available online at www.jafmonline.net, ISSN 1735-3572, EISSN 1735-3645.

DOI: $10.36884 / \mathrm{jafm} .9$. SI2.25793

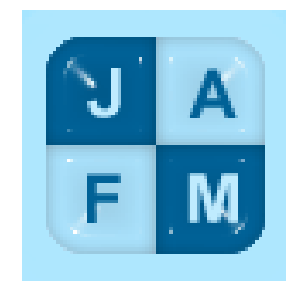

\title{
Fuzzy Kalman Filtering for 3-D Lagrangian Particle Tracking using Blob Detection
}

\author{
S. Rezig ${ }^{1 \dagger}$, R. Toscano ${ }^{1}$, G. Rusaouën ${ }^{2}$ and V. Lozano ${ }^{1}$ \\ ${ }^{1}$ LTDS, Ecole d'ingénieurs de Saint-Etienne (ENISE), Saint-Etienne, France \\ ${ }^{2}$ CETHIL, Institut National des Sciences Appliquées, Lyon, France \\ †Corresponding Author Email: sawsen.rezig@enise.fr
}

(Received October 8, 2015; accepted December 10, 2015)

\begin{abstract}
3-D Lagrangian Particle Tracking (3DLPT) is becoming widely used to characterize the convective indoor air movements in large scale spaces. The need to implement a robust algorithm led us to develop a multi-scale based approach to detect features (Helium filled soap bubbles). On the other hand, the particle tracking is another challenging problem. To this end, a new tracking algorithm based on fuzzy Kalman filtering is proposed in this paper. The Kalman filter is used to optimally estimate the new position of the particles based on their actual position. In our approach, the initial particle positions are represented with multivariate fuzzy sets.
\end{abstract}

Keywords: Indoor airflow; 3D PTV; Particle detection; Temporal tracking; Kalman filtering; Fuzzy logic.

\section{NOMENCLATURE}

$\begin{array}{ll}d^{E} & \text { random vector for process noise } \\ d^{m} & \text { random vector for measurement noise } \\ F K F & \text { Fuzzy Kalman Filter } \\ K & \text { optimal Kalman gain } \\ k & \text { discrete time instant } \\ M & \text { particle mass } \\ m(k) & \text { measurement at the time instant } k\end{array}$

\section{INTRODUCTION}

A critically important quantity of energy is required nowadays to heat and ventilate our buildings. In order to reduce this energy demand, we need to develop robust diagnostic tools for air flows in large scale spaces like buildings rooms. Particle image velocimetry (PIV) algorithms using crosscorrelation of particle images at two time steps can be used to obtain quantitative information of the airflow velocity field; it enables to calculate quantities like vorticity and deformation. However, they still limited when investigating strong gradients (Kähler et al. 2012). The velocity estimation is performed within an interrogation window; the required size of interrogation window depends on the particle size and the seeding density (Raffel et al. 2007), this leads to errors when particle density changes. Bubble tracking using PIV was studied in (Cheng et al. 2005), it was concluded that it is not applicable in dense bubbly

$\begin{array}{ll}N & \text { number of particles at time instant } t \\ p_{i} & \text { particle } \mathrm{i} \\ Q & \text { process noise covariance matrix } \\ R & \text { measurement noise covariance matrix } \\ T_{e} & \text { time step } \\ \varepsilon(k) & \text { state vector at time instant } k\end{array}$

flows as long as bubbles overlap.

In order to obtain spatially resolved velocity measurement, Lagrangian particle tracking (LPT) and particle tracking velocimetry (PTV) techniques are used; PTV algorithms have two principal advantages with respect to classical PIV. First, they are able to provide three-dimensional (3D) flows. Second, Eulerian and Lagrangian statistics may be calculated from the Lagrangian-type PTV output; however, the calculation of Lagrangian statistics from a regular Eulerian data set, as obtained with PIV, is less accurate.

The use of 3D PTV requires identifying the helium filled soap bubbles on the recorded images and then reconstructing their $3 \mathrm{D}$ trajectories via stereoscopic analysis. In this paper, the emphasis is on two dominant error sources:

1. Particle positioning: errors related to determination of particle positions.

2. Image pairing: errors due to false particle 
matching.

Noise present in recorded images, false detections and overlapping particles lead to not fully reconstructed trajectories, the performance of the tracking algorithm is hence widely influenced by the quality of particle detection results at high seeding concentrations. Using more than two cameras allows compensating the loss of precision; we designed here a feedback schema which uses multi-view information to correct uncertainty in particle positioning (Fig. 1).

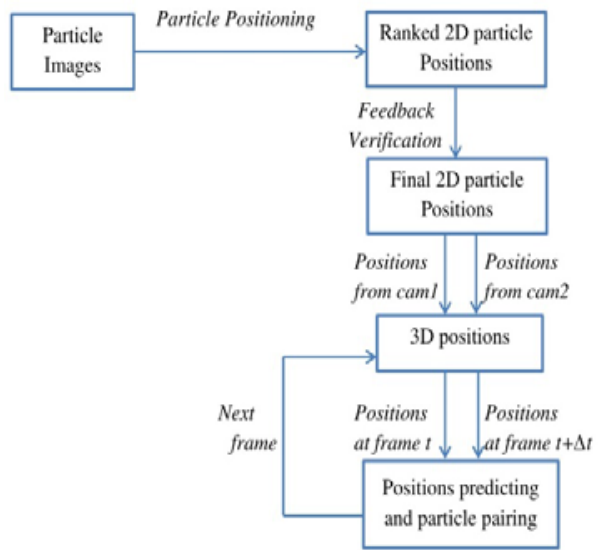

Fig. 1. A Global schema illustrating the proposed feedback approach.

Bubble detection consists of analyzing the recorded particle images and calculating the bary-center of each particle on the image. The simplest manner to achieve this is to threshold the image by defining an intensity level to separate pixels belonging to particles and pixels of background, this binarization of the image can be performed automatically (Otsu et al. (1979)) by minimizing the interclass-variance of the separated classes of pixels; although this is intuitive, a big loss of information is engendered by such methods due to noise and illumination issues as only intensity is considered and not any other feature proprieties. Techniques like calculation of the optical flow equation (Shindler et al. 2010) and pattern recognition (Takehara et al. 2000) were proposed. These methods detects peaks in grey level intensity but still too sensitive to noise. Neural network particle algorithm was proposed to improve accuracy when the images are noisy Ouellette et al. (2006). Three-pinhole defocusing concept was employed in Yoon et al. (2006) for micro PIV to detect particles, yet low light intensity caused by pinholes is a drawback of the developed method, furthermore, the calibration step have to be repeated for different fluids.

On the other hand, the particle pairing is another challenging problem. The particle image information acquired at each time steps is used for a reliable particle pairing, in PTV study case the particles used are identical soap bubbles filled with helium, this makes the reconstruction of particle trajectories particularly difficult. In an attempt to remedy this, a nearest neighbor algorithm was first used (Malik et al. 1993); this approach works only at low densities. Alternatively, (Pereira et al. 2006) investigated neural networks and relaxation methods, relaxation schema was found to be robust and much faster than neural networks. The prototype of multi-frame tracking algorithms was described in Guezennec et al. (1994). Ouellette et al. (2006) and Cierpka et al. (2013) proposed then 4-frame predictive algorithms for 3D PTV using extrapolation based on proprieties of the flow like acceleration and velocity. Regression instead of the extrapolation was later employed (Biwole et al. 2009, Li et al. 2008) to recover longer trajectories. Temporal tracking algorithms were developed based on polynomial. Cardwell et al. (2011) employed a variable pair-matching algorithm which utilizes displacement preconditioning and estimated particle proprieties. Suziki et al. (2012) algorithm optimizes the data input temporally and spatially by introducing a reduced-order Kalman filter. Several other tracking techniques such as the combined extended Kalman filter and nearest neighbor standard filter approach was described in Straw et al. (2010).

Kalman filtering was recently used in this field to predict the particle trajectory in PIV algorithm (Suzuki et al. 2012). While these works are devoted to PIV methods and generally only small-scale 3D flows, this paper highlights the problem of enhancing performance of temporal tracking for 3D PTV methodology; a new tracking algorithm based on fuzzy Kalman filtering and is proposed herein.

\section{OUR METhOD}

In what follows, the basic principles of our approach is presented as well as the numerical experiments, which show accurately determined and tracked particles in a stable manner over time.

\subsection{Multi-scale Particle Positioning}

The recorded images include particles of variable sizes; in fact, in the case of PTV the injected particles are $1.3 \mathrm{~mm}-3.8 \mathrm{~mm}$ helium filled soap bubbles, also, their size depends on their distance from the camera. On the other hand, intensity and shape of particles pixels vary with the way particles are enlightened. This makes particle detections step a challenging step, besides, a precise positioning of the tracked bubbles significantly enhances matching process functioning. In this case, a simple barycenter calculation is not efficient due to overlapping problem.

Using more than two cameras enables us to compensate the loss of precision; hence we designed a feedback schema witch uses multi-view information to correct uncertainty in particle positioning. The particles detected by our multiscale method are classified according to their reliability; two classes of responses were defined:

1. One-view particles: isolated and well viewed particles are detected by the method as one position corresponding to their barycenter, in these cases no verification is needed and 
positions are tagged a detected particles.

2. Two-view particles: isolated bright pixels are considered as potential particles, they may be due to poorly enlightened bubbles or bubbles situated on the border of the observed area, in these cases we need to verify if the bubble is visible bye other cameras, if not it is concluded that the pixel is aberrant.

These positions are used as feedback information; it allows correcting the first iteration of bubble positioning. At the second iteration, only the positions classified at least once as One-view particles will be kept.

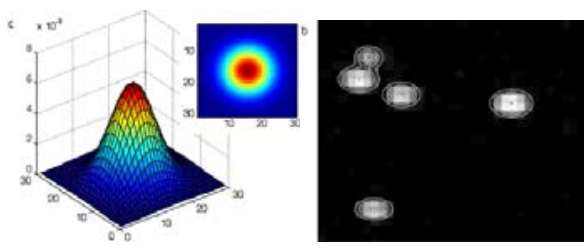

Fig. 2. (3D/2D) Gaussian function compared to particle image.

The need to implement robust detection algorithm led us to improve our method by developing a multi-scale based approach to detect features, for this purpose, we employed a pattern recognition technique using the cross-correlation between artificial patterns close to the real particle appearance and an interrogation region in the particle image plane. The prototype of this method is described in Mann et al. (2000), here, a particle pattern approximates an "ideal" particle image as a two dimensional Gaussian distribution template with a standard deviation $\delta$, see Fig. 2 .

Furthermore, the particles partially overlapped (see Fig. 2), this constitutes regions of bright pixels with a shape resulting from adding two or more Gaussian functions. Unlike isolated particles, overlapping particles cannot be detected when scanning image using a Gaussian pattern. Thus it is clear to see that a blob detection technique is more suitable to our problem. These mathematical methods aim to extract regions in image that differ in brightness, color and other properties, in fact these regions of interest, also known as blobs, have a high degree of variation in all directions hence the values of their pixels vary according to well defined functions (a two dimensional Gaussian distribution in our study case as concluded before).

\subsection{Fuzzy Kalman Filter for temporal tracking}

In this section, it is shown that the particle image information acquired at each time step can be used for a reliable particle pairing and tracking by introducing a new kind of estimator called the Fuzzy Kalman Filter (FKF). The Kalman filter is used to optimally estimate the new position of the particles based on their actual positions, this is done through a simple mechanical model of the particles evolution combined with a measurement process. The initialization step is usually performed via the first image camera samples. However, some uncertainties are always present. In our approach, this is taken into account by representing the initial particle positions with multivariate fuzzy sets. In this way, the algorithm starts in a state of fuzzy knowledge about the particle matching; but, as we accumulate information through the measurement process, we improve the particles location estimate.

In what follows, some basic principles of the standard Kalman filter are given and the extended to its fuzzy version in the context of particle tracking.

Roughly speaking, a Kalman filter is an optimal recursive data processing algorithm (Maybeck 1979). The optimality must be understood as the best estimate which we can make according to the model used for the measurement process as well as the data used to compute; this estimate it is based on the state-space model describing the evolution of the system of interest. These state-space and measurement models are represented by the following linear discrete stochastic equations

$\left\{\begin{array}{l}\varepsilon(k+1)=F \varepsilon(k)+d^{\varepsilon}(k) \\ m(k)=C \varepsilon(k)+d^{m}(k)\end{array}\right.$

The noise vectors are assumed to be independent (of each other), white, and with normal probability distributions $d^{E}(k) \sim \mathrm{N}(0, \mathrm{Q}), d^{m}(k) \sim \mathrm{N}(0, \mathrm{R})$. These matrices are assumed to be known, in fact as we will see, $\mathrm{Q}$ and $\mathrm{R}$ can be be used as tuning parameter of the Kalman filter. The objective is to design an optimal estimator which combines a prior estimation of $\varepsilon(k)$ and the measurement $m(k)$, so that the resulting posterior estimate is optimal in a sense which will be defined further.

The Kalman filter is then given by:

$$
\left\{\begin{array}{l}
\hat{\varepsilon}(k+1)=F(I-K C) \hat{\varepsilon}(k)+F K m(k) \\
\hat{m}(k)=C \hat{\varepsilon}(k)
\end{array}\right.
$$

The optimal Kalman gain $K$ is given by the stationary solution of a Ricatti equation obtained when the aim of the optimization is that the expectation of the posterior estimation error is zero and its variance is minimal. In what follows we present a guideline on the choice of the matrices Q and $\mathrm{R}$.

As said before, the matrices $\mathrm{Q}$ and $\mathrm{R}$ can be adjusted by the user according to the confidence placed in the prediction or measurement phase. When the largest eigenvalue of $\mathrm{Q}$ is very small compared to smallest eigenvalue of $\mathrm{R}$ this mean that a great importance is accorded to the prediction phase, in other words we are confident with the precision of the model used to represents the system under study. This means also that we are not very confident with the measurement process. When the 
smallest eigenvalue of $\mathrm{Q}$ is big compared to the largest eigenvalue of $\mathrm{R}$, this mean that a small importance is accorded to the prediction phase, in other words we are not too much confident with the accuracy of the model and a great importance is accorded to the measurement process.

The figure Fig. 3 illustrates, in two dimensions, the problem of particle tracking: knowing the coordinates of the particle $p_{i}$ at the time instant $k$, where is this particle at the time instant $k+1$ ? The knowledge of the coordinate of the particle at any time allows constructing the trajectory followed by the particle.

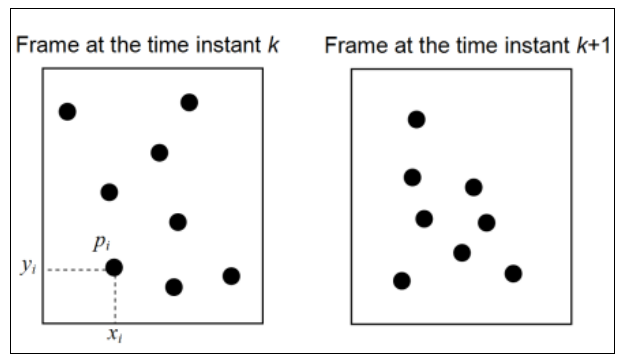

Fig. 3. 2D representation of the particles image for two successive time instants. The coordinate of the particle pi is known at time instant $k$ but where is the particle pi at the time instant $k+1$ ?

This is the problem of particle tracking which is not at all obvious notably when the density of

particle is high.

The solution to this problem is not at all obvious notably when the density of the particle is high and/or the sampling time $T e$ is high. To solve this problem used a Kalman filter with a simple mechanical model of the particle evolution combined with a measurement process.

The position of a particle $p_{i}$ is defined by its spatial coordinates: $p_{i}(t)=\left(x_{i}(t), y_{i}(t), z_{i}(t)\right)^{T}$. The velocity of the particle is given by its time derivative $d / d t\left(\left(x_{i}(t)\right.\right.$, $\left.y_{i}(t), z_{i}(t)\right)^{T}=\left(v_{x i}(t), v_{y i}(t), v_{z i}(t)\right)^{T}$. By application of the fundamental principle of the dynamic we get the equation of motion of a particle $\mathrm{p}_{\mathrm{i}}$ according to one direction, say $\mathrm{x}$-direction:

$\left[\begin{array}{l}\dot{x}_{i}(t) \\ \dot{v}_{x i}(t)\end{array}\right]=\left[\begin{array}{ll}0 & 1 \\ 0 & 0\end{array}\right]\left[\begin{array}{l}x_{i}(t) \\ v_{x i}(t)\end{array}\right]+\left[\begin{array}{l}0 \\ 1\end{array}\right] F_{x i}(t) / M$

In equation (3), $F_{x i}(t)$ is the unknown force acting on the particle $p_{i}$ for $\mathrm{x}$-direction.

The same equations are applied for the other directions and are omitted for the simplicity of the exposition

To make use of the Kalman filter we have to discretize the continuous time equation of movement (3):

$\left.\left[\begin{array}{l}x_{i}(k+1) \\ v_{x i}(k+1)\end{array}\right]=\left[\begin{array}{cc}1 & T_{e} \\ 0 & 1\end{array}\right] \begin{array}{l}x_{i}(k) \\ v_{x i}(k)\end{array}\right]+\left[\begin{array}{l}1 / 2 T_{e} \\ T_{e}\end{array}\right] F_{x i}(k) / M$

The complete dynamic equation including the dynamic evolution of the state vector together with the measurement process is then given by:
$\left\{\begin{array}{l}\varepsilon_{i}(k+1)=F \varepsilon_{i}(k)+d^{\varepsilon_{i}}(k) \\ m_{i}^{\varepsilon}(k)=C \varepsilon_{i}(k)+d_{i}^{m}(k)\end{array}\right.$

Which is in the required form to apply the Kalman filter

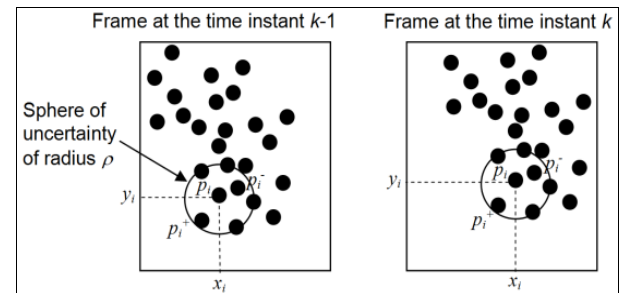

Fig. 4. The initialization procedure is realized from the first frames taken at the time instant $k=0$ and $k=1$. The particle pairing is based on a proximity criterion.

\subsection{Initialization of the Kalman temporal tracking algorithm}

As shown in the figure Fig. 4., to take into account the uncertainty in the particle pairing, we consider around each particle $p_{i}(k-1)$ a sphere of uncertainty of radius $\rho$. For $\rho=0$ we recover the Kalman particle tracking described above. We associate to $p_{i}(k-1)$ the two near particles denoted $p_{i}^{+}(k-1)$ and $p_{i}^{-}(k-1)$, we can then define for each direction an interval of uncertainty. For instance, in the $\mathrm{x}$-direction the intervals of uncertainties $I_{x}^{i}(k-1)$ and $I_{v x}^{i}(k-1)$ are defined between the minimum and maximum of coordinates and velocities of $p_{i}(k-1), p_{i}^{+}(k-1)$ and $p_{i}^{-}$ $(k-1)$.

We will assume that the real position and velocity of the particle $\mathrm{p}_{\mathrm{i}}$ is distributed with a confidence function over the intervals $I_{x}^{i}(k-1)$ and $I_{v x}^{i}(k-1)$ respectively. In what follows, the confidence function will be referred to as membership function associated to some fuzzy sets. Here, three fuzzy sets are associated to the interval $I_{x}^{i}(k-1)$ namely: Lower, Medium and Upper (see Fig. 5). The same fuzzy partition is associated to $I_{v x}^{i}(k-1)$, it is omitted here for simplicity.

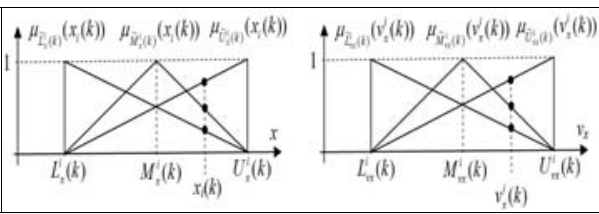

Fig. 5. Fuzzy sets associated to the interval $I_{x}^{i}(k-$ 1) and $I_{v x}^{i}(k-1)$.

According to what has been said before, the fuzzy Kalman filter is defined via a fuzzy rule base associated to the fuzzy partition defined above. Note that this fuzzy Kalman filter is similar to the Takagi-Sugeno fuzzy model adopted fuzzy control area. However our approach is used in an entirely different context and has nothing to do with fuzzy modelling. With this fuzzy Kalman filter three predictions are weighted by the center of the uncertainty sphere. The final prediction is realized 
by a weighted average of these three predictions (standard defuzzification).

\section{VALIDATION}

Two data sets were chosen here to validate our approach; these benchmarks fit our purpose of investigation very well, they provide $3 \mathrm{D}$ flow trajectories of various lengths:

1. Synthetic data: Data set 352 are from Standard PIV Images project developed by the Visualization Society of Japan (Okamoto et al. (2000)), this describes a jet flow impinging on a wall, about 300 particles were observed at each time frame.

2. Experimental data: This was provided by [1], it describes a particle flow composed of 310 trajectories observed during 200 frames.

Table 1 FKF application results

\begin{tabular}{|c|c|c|}
\hline Data set & $\begin{array}{c}\text { Correct } \\
\text { ratio }\end{array}$ & $\begin{array}{c}\text { Correct } \\
\text { trajectory } \\
\text { ratio }\end{array}$ \\
\hline $\begin{array}{c}\text { VSJ data } \\
\text { set 352 }\end{array}$ & 0.98 & 0.94 \\
\hline $\begin{array}{c}\text { Wesleyan } \\
\text { U. data set }\end{array}$ & 0.96 & 0.92 \\
\hline
\end{tabular}

The proposed FKF is used to track particles during their displacement; it has been applied to the synthetic and experimental data, table 1 . shows, for each data set, the "correct ratio" described in Li et al. (2008), this ratio shows the percentage of correctly tracked particle images, the ratio of the number of correct tracked trajectories divided by the total number of input was calculated as well.

The detection error is calculated for both data sets, figure Fig. 6 shows the distribution of this error versus the length of particles in the image, the detection algorithm parameters were fixed for each data set as follows:

1. The scale interval $A=[0.25,1.25]$ for $\mathrm{LoG}$ based calculation and $\alpha=0.25$ for Hessian based extraction.

2. The scale interval $A=[0.3,1.3]$ for $\mathrm{LoG}$ based calculation and $\alpha=0.3$ for Hessian based extraction.

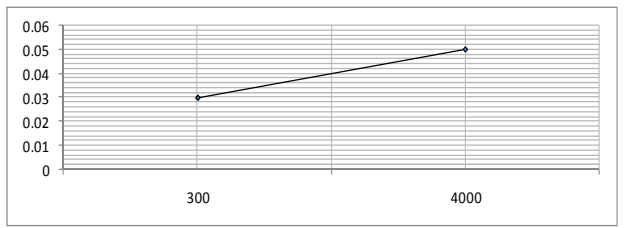

Fig. 6. Distribution of the detection process error.

\section{Conclusion}

A new FKF based algorithm is proposed here for particle tracking, it was also designed to take into account uncertainties at initial state and errors due to bad bubbles positioning are efficiently avoided using a modified multi-scale blob detection approach combining two different measures.

\section{ACKNOWLEDGEMENTS}

This work was funded by the ANR (French National Agency for Research) as part of the ANR12-VBDU-0010 project FluBat (Monitoring and experimental understanding of airflow in the buildings).

\section{REFERENCES}

Biwole, P. H., W. Yan, Y. Zhang, and J. J. Roux (2009). A complete 3D particle tracking algorithm and its applications to the indoor airflow study. Meas Sci Tech 20(11).

Cardwell, N. D., P. P. Vlachos, and K. A. Thole (2011). A multi-parametric particle-pairing algorithm for particle tracking in single and multiphase flows. Meas. Sci. Tech 22(10), 105406.

Cheng, W., Y. Murai, T. Sasaki, and F. Yamamoto (2005). Bubble velocity measurement with a recursive cross correlation PIV technique Flow. Flow Meas Ins 16(1),1635-1646.

Cierpka, C., B. Lütke, and C. J. Kähler (2013). Higher order multi-frame particle tracking velocimetry, Exp Fluids 54(5).

Guezennec, Y. G., R. S. Brodkey, N. Trigui, and J. C. Kent (1994). Algorithms for fully automated three dimensional particle tracking velocimetry. Exp. Fluids 17(4),209-219.

Kähler, C. J., S. Scharnowski, and C. Cierpka (2012). On the resolution limit of digital particle image velocimetry. Exp. Fluids 52(6),1629-1639.

Li, D., Y. Zhang, Y. Sun, and W. Yan (2008). A multi-frame particle tracking algorithm robust against input noise. Meas Sci Tech 19(10).

Malik, N., T. Dracos, and D. Papantoniou (1993), Particle Tracking in three dimensional turbulent flows. Exp. Fluids 15(2),279-294.

Mann, J., S. Ott, and J. S. Andersen (1999). Experimental study of relative, turbulent diffusion. Risø National Laboratory Report Risø-R-1036.

Maybeck, P.S. (1979), Stochastic models, estimation, and control. Academic Press.

Okamoto, K., S. Nishio, T. Kobayashi, T. Saga, and K. Takehara (2000), Evaluation of the 3DPIV Standard Images (PIV-STD Project). $J$. Visualization 3(2),115-124. 
S. Rezig et al. / JAFM, Vol. 9, Special Issue 2, pp. 207-212, 2016.

Otsu, N. (1979). A threshold selection method from gray-level histograms. IEEE Trans on Syst, Man Cybern 9(1),62-66.

Ouellette, N. T., H. Xu, and E. Bodenschatz (2006) A quantitative study of three-dimensional Lagrangian particle tracking algorithms. Exp Fluids 40(2),301-313.

Pereira, F., H. Stuer, E.C. Graft, and M. Gharib (2006). Two-frame 3d particle tracking. Meas Sci Tech 17(7),1680-92.

Raffel, M., C. E. Willert, S. T. Wereley, and J. Kompenhans (2007). Particle image velocimetry. A practical guide. 2 nd ed. Berlin.: Springer.

Shindler, L., M. Moroni, and A. Cenedese (2010) Spatial-temporal improvements of a two-frame particle-tracking algorithm. Meas Sci Tech
21(11), 115-401.

Straw, A. D., K. Branson, T.R. Neumann, and M.H. Dickinson (2010). Multi-camera realtime 3d tracking of multiple flying animals. $J$. of The Royal Society Interface, rsif20100230.

Suzuki, T. (2012). Reduced-order Kalman-filtered hybrid simulation combining article tracking velocimetry and direct numerical simulation. $J$. of Fluid Mech 709(10), 249-288.

Takehara, K., R. J. Adrian, G. T. Etoh, and K. T Christense (2000). A Kalman tracker for superresolution PIV. Exp. Fluids 29(1), 34-41.

Yoon, S. Y., and K. C. Kim (2006). 3D particle position and $3 \mathrm{D}$ velocity field measurement in a micro-volume via the defocusing concept. Meas. Sci. Tech 17(11),897-905. 